\title{
The Time Evolution of the Radio Supernovae in Arp 220
}

\author{
Colin J. Lonsdale \\ MIT Haystack Observatory, Westford MA 01886, USA
}

Philip J. Diamond

Jodrell Bank Observatory, Jodrell Bank, Cheshire SK11 9DL

Carol J. Lonsdale

IPAC, MS 100-22, 770 S. Wilson Ave., Pasadena, CA 91125, USA

Harding E. Smith

UCSD CASS, 9500 Gilman Dr., La Jolla, CA 92093-0424, USA

\begin{abstract}
Since our unexpected discovery of 12 compact radio sources in one of the nuclei of Arp220 we have been monitoring the structure using global VLBI and the VLBA. With several epochs of data we can now demonstrate that the fluxes of the radio sources are, in general, decreasing sytematically but not smoothly. The sources also have steep spectra. Both facts support our earlier supposition that these are highly luminous radio supernovae. We initially predicted a radio supernova rate of approximately $2 y^{-1}$. Our most recent observations indicate that the occurrence rate of luminous RSN is several times lower than predicted, thus our model of the Arp220 starburst must be revised.
\end{abstract}

\section{Introduction}

The phenomenon of luminous infrared galaxies is clearly related to galactic collision and merger events. Large quantities of gas and dust are robbed of angular momentum with respect to the merging system, and fall into the central regions, creating a dense and highly opaque nuclear condensation, often only a few hundred parsecs in size. During this process, a powerful energy source is created, but hidden from direct view, inside the nuclear condensation. Most of the bolometric luminosity emerges in the mid and far infrared regions of the spectrum after extensive dust reprocessing. Evidence is strong that such systems evolve, a process which results in gradual clearing of the nuclear dust shroud, and at some point leads to the unveiling of a luminous AGN, unambiguously fuelled by accretion onto a supermassive black hole.

Many uncertainties still surround this process. Do all merging systems eventually create a luminous AGN? Is the power source during the enshrouded phase a massive nuclear starburst, a hidden AGN, or some combination? How 
strong are orientation effects due to anisotropic obscuration? Answering such questions is difficult because the nucleus is opaque at most wavelengths, and few observing techniques offer sufficient angular resolution to yield more than one pixel across the emitting region.

For these reasons, we have been pursuing a program of sensitive VLBI observations of luminous IR galaxies, at $18 \mathrm{~cm}$ where the optical depths to the nuclear regions are small. Such observations yield hundreds of resolution elements across the nuclear regions of these galaxies, offering the prospect of detailed information concerning the nature and evolution of that energy source. The common detection of strong, high brightness temperature emission in these galaxies suggests the presence of AGN sources in the majority of luminous IR galaxies. Although estimation of the bolometric luminosity of such AGN sources is perilous, our results are consistent with a buried AGN origin for a major portion of the total luminosity of such systems (Lonsdale, Smith \& Lonsdale 1995)

In the prototypical luminous IR galaxy Arp 220, however, our data reveal a remarkable cluster of unresolved sources distributed throughout the nuclear region (Smith et al. 1998). Interpreting these sources as extraordinarily luminous radio supernovae in an intense nuclear starburst, we have initiated a monitoring campaign of the RSN light curves. In this contribution, we briefly describe the results of our monitoring observations of Arp 220, and the implications for the nature of the RSN, their surroundings, and of the nuclear activity in Arp 220.

\section{Observations and Results}

All our imaging VLBI observations of Arp 220 have been conducted using a VLBI array involving the VLBA, plus one or more sensitive telescopes. The early observations included large EVN telescopes, while the later, regular monitoring observations involved just the VLBA and phased VLA. The continuum sources in Arp 220 are too weak for effective self-calibration, so we employ a spectral phase referencing technique, utilizing a strong, compact $\mathrm{OH}$ megamaser feature in Arp 220, as described by Lonsdale et al. (1998). The image shown in Figure 1 is typical of the results we obtain from an epoch of VLBA plus phased VLA observations. The noise level is on the order of $0.08 \mathrm{mJy} / \mathrm{beam}$, and the angular resolution is roughly 5 millarcsec $(1.5 \mathrm{pc})$ in the $\mathrm{E}-\mathrm{W}$ direction. The image is centered on the western nucleus of Arp 220. The eastern nucleus is roughly 1 arcsec away, and contains 2 or 3 weak point sources, not shown here. All of the sources are unresolved (smaller than 1 parsec).

The most important result from the monitoring is the appearance of two new sources since 1994 , implying an occurrence rate of roughly $0.3 / \mathrm{yr}$. Confirmation of these two sources was obtained from the June 2000 observations, but careful inspection of previous epochs clearly shows the existence of one source nearly 3 years earlier. Based on the number of sources present, one can estimate a source lifetime, above the detection threshold of $0.25 \mathrm{mJy}$, of order 30 years. This is consistent with the observed mean rate of decline of flux density for the sources. Fluctuations of flux density of $30-50 \%$ are evident on timescales of 1 year for some sources, however. 


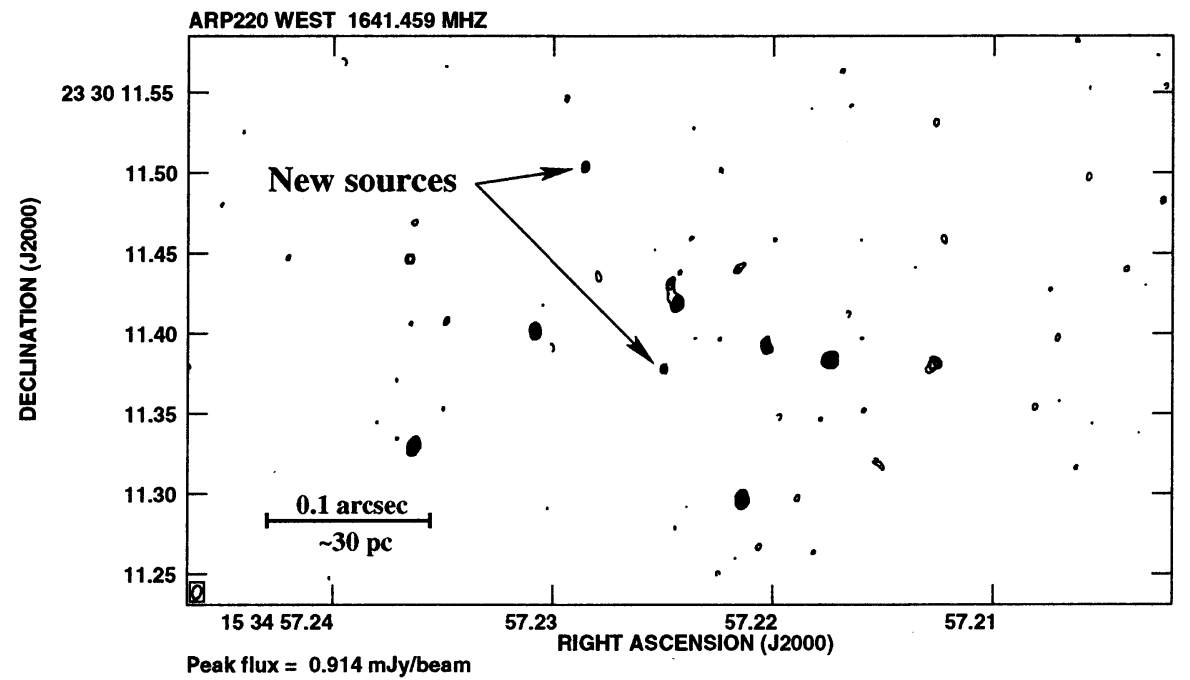

Figure 1. The western nucleus of Arp 220 at $18 \mathrm{~cm}$, in June 2000. Two new sources, indicated by arrows, have appeared since 1994 .

\section{Interpretation as Radio Supernovae}

The compact continuum radio sources in Arp 220 do not behave in a manner typical of radio supernovae. Their apparent light curves do not conform to the well-established RSN pattern of a rapid rise, followed by a smooth exponential decay with an e-folding time of a few years. In addition, each of the detected objects has a radio luminosity at the extreme upper end of the distribution of known RSN luminosities. A natural question to ask is therefore whether they are radio supernovae at all. We argue that radio supernovae remain the most plausible explanation for these sources, for a variety of reasons.

The sources are sub-parsec, varying substantially on timescales of months, indicating emitting regions of a scale consistent with the RSN phenomenon. Steep radio spectra indicate synchrotron radiation like that responsible for conventional RSN. The general decline in flux density of the ensemble of sources, plus the appearance of 2 new sources in 6 years, places these objects in a class of short-lived phenomena to which supernovae belong. RSN light curves and luminosities are thought to be largely dictated by interaction of the supernova blast wave with the pre-existing circumstellar environment, normally the progenitor stellar wind (Chevalier 1994). In the Arp 220 nuclear environment, however, the medium into which the supernova explodes may be much denser and more inhomogeneous than that leading to conventional RSN. The resulting RSN may exhibit a radio light curve of longer duration and greater luminosity, and with larger fluctuations, than is seen in normal RSN. Finally, the starbursting nucleus of Arp 220 is, arguably, the most likely place in the nearby Universe for a cluster of bright supernovae to occur. The apparently random sprinkling of sources across the densest regions of the nucleus closely echoes simple expectations for 
the spatial distribution of supernova events in this object. While we are aware that the above represent only consistency and plausibility arguments, the weight of evidence in favor of a radio supernova interpretation is compelling.

\section{Implications and Conclusions}

A promising approach to improved understanding the starburst which gave rise to the progenitor stars, and the dense environment into which the supernovae are exploding is to correlate RSN-producing regions with information from other starburst and AGN tracers. Using forthcoming mm-wavelength molecular line imaging capabilities, the density, temperature and chemistry of the starbursting region can be mapped in useful detail. Knowledge that regions with particular characteristics are forming massive supernova progenitors in large numbers may lead to valuable insights. Our own studies of the $\mathrm{OH}$ masing regions already provide tantalizing clues, with suggestions that starburst-generated shocks trigger the masers. A critical issue is identifying and locating an AGN source in Arp 220, if one exists. The disposition of such an AGN with respect to the RSN-traced starburst regions may provide clues to the relationship between the two phenomena in these objects.

Our RSN data can be used to improve our understanding of the RSN phenomenon itself. One interpretation of the large variations in flux density on 1year timescales that we observe is that a normal $10,000 \mathrm{~km} / \mathrm{sec}$ supernova shock wave is encountering strong inhomogeneity in the circumstellar environment, on scales of 0.01 parsec. The observed slow rising phase of the light curve suggests that the cavity generated by the progenitor wind is highly non-spherical, so that dense material is gradually encountered some time after the initial explosion, ushering in a long-lived anomalously luminous phase unseen in normal RSN.

Continued monitoring of the Arp $220 \mathrm{RSN}$ will yield important and fascinating information about the obscured inner nuclear regions of this galaxy. We will shortly be conducting observations of considerably higher sensitivity, which will sample the RSN luminosity function to much fainter levels. We will also gain much more detailed knowledge of the spatial relationship between the RSN and the $\mathrm{OH}$ maser regions. The possibility exists that an unambiguous AGN signature will emerge. As VLBI sensitivities improve, observations similar to those we are conducting on Ȧrp 220 will become feasible for more distant objects, and the prospects for this line of research making key contributions to our understanding of galaxy evolution, mergers and nuclear activity are excellent.

\section{References}

Chevalier, R. ApJ, 420, 268.

Lonsdale, C., Diamond, P., Lonsdale, C. \& Smith, H. E. 1998, ApJ, 493, L13.

Lonsdale, C., Smith, H. E., \& Lonsdale, C. 1995, ApJ, 438, 632.

Smith, H. E., Lonsdale, C., Lonsdale, C. \& Diamond, P. 1998, ApJ, 493, L17. 